\title{
Effective methods of pelvic plexus nerve and bladder stimulation in anesthetized animal model
}

\author{
Larissa Bresler, MD; ${ }^{1-2}$ James S. Walter, PhD; ${ }^{2-3^{*}}$ Andrew Jahoda, MD; ${ }^{1-2}$ John S. Wheeler, MD; ${ }^{1-2}$ Thomas \\ Turk, MD; ${ }^{1-2}$ Robert D. Wurster, PhD ${ }^{2,4}$ \\ ${ }^{1}$ Section of Urology, Edward Hines Jr Department of Veterans Affairs (VA) Hospital, Hines, IL; ${ }^{2}$ Department of Urology, \\ Stritch School of Medicine, Loyola University Chicago, Maywood, IL; ${ }^{3}$ Research Service, Edward Hines Jr VA Hospital, \\ Hines, IL; ${ }^{4}$ Departments of Neurological Surgery and Physiology, Stritch School of Medicine, Loyola University Chicago, \\ Maywood, IL
}

\begin{abstract}
Urinary retention is a serious urological problem associated with spinal cord injuries (SCIs) and other pelvic disorders. Effective methods of pelvic nerve stimulation were investigated for this problem. Following anesthesia in five dogs, the bladder was surgically exposed. Bladder and anal sphincter pressures were recorded. Testing was first conducted with probe electrodes. Barb electrodes were then implanted with a needle near the pelvic plexus nerves and the bladder wall. We tested different electrode arrangements and stimulating parameters to induce bladder contractions without skeletal muscle stimulation. The pelvic plexus nerves near the bladder were identified, and the barb electrodes were effectively implanted. Stimulation with bipolar and bilateral electrodes induced pressures over $30 \mathrm{cmH}_{2} \mathrm{O}$ without skeletal muscle activation. Common stimulation parameters were $40 \mathrm{pps}, 400$ microseconds pulse duration, and 15 to $25 \mathrm{~mA}$ stimulating current applied for $3 \mathrm{~s}$. Effective electrode implantation methods were shown. Also identified were electrode arrangements and stimulating parameters that induced strong bladder contractions without skeletal muscle activation. However, voiding studies were not conducted. Further studies with barb electrodes are warranted, and these methods may have applications for bladder stimulation following SCI.
\end{abstract}

Key words: dog, electrical stimulation, pelvic plexus nerves, spinal cord injuries, urinary bladder, urinary incontinence, urinary retention, urinary tract physiology, urination disorders, urodynamic.

\section{INTRODUCTION}

Urinary retention is a serious urological problem associated with spinal cord injuries (SCIs) and pelvic surgeries, or it may be idiopathic [1-4]. Electrical stimulation has been investigated as a method to promote bladder contraction and bladder emptying. This approach has benefited individuals with SCI who have urinary retention. The "Brindley method" is the best known use of electrical stimulation and includes electrodes around the sacral nerves within the sacrum [5-6]. However, this method includes invasive spinal surgery and a sacral nerve afferent neurectomy. Alternatively, direct bladder stimulation with electrodes implanted near the pelvic plexus nerves and bladder wall has been investigated [7-8].

Direct bladder stimulation in the patient with SCI requires high stimulation current for inducing bladder contractions and voiding [9-15]. High current is a problem because the spread of the electrical current can stimulate

\footnotetext{
Abbreviation: SCI = spinal cord injury.

*Address all correspondence to James S. Walter, PhD; Edward Hines Jr VA Hospital, Research Service, Bldg 1, Rm C-318 (151), 5000 South 5th Ave, Hines, IL 60141; 708202-5805; fax: 708-202-2319. Email:james.walter@va.gov DOI: 10.1682/JRRD.2007.04.0058
} 
legs and urethral sphincters [9-13]. This problem is difficult because the pelvic plexus nerves innervating the bladder contain $\delta$ and $\mathrm{C}$ fibers that are small and require higher current for activation than the larger fibers innervating skeletal muscles [12]. Neuroprosthetic methods that can be used for stimulating the pelvic plexus nerves at low currents to limit the spread of the electrical currents are thus an important area of investigation. Approaches include (1) small electrodes that have high charge injection density [16-17], (2) a narrow separation of bipolar electrodes for reducing the spread of the electric current [18], (3) standard neurosurgical procedures of nerve identification and implantation of electrodes close to the nerves [16-17], and (4) high stimulating frequencies and long pulse durations that may induce bladder contractions at lower stimulating currents [19-21]. In addition, the introduction of the Peterson Intramuscular Electrode (Synapse Biomedical; Oberlin, Ohio) device may facilitate some of these approaches [17]. This electrode features a small surface area and implantation with a needle that facilitates both implanting electrodes close together and close to a nerve.

In this investigation, a barb electrode similar to the Peterson electrode was used. The barb electrode had insulated wire except for the stimulating tip and was implanted with a needle. We used an acute animal model with a respiratory anesthetic that partially suppresses bladder reflexes to better show effects of pelvic plexus nerve stimulation. We investigated different electrode arrangements and stimulating parameters to try to induce bladder contractions without skeletal muscle activation.

\section{METHODS}

Three different types of electrodes were used in this study: probe, barb, and woven eye (Figure 1). A commercial barb-type electrode, the Peterson Intramuscular Electrode, is also shown in Figure 1 [16-17]; however, it was not used in this study but is shown here for comparison. For initial testing, the probe electrode was made from a nerve block needle (HN1, Professional Instruments; Houston, Texas). The last $8 \mathrm{~mm}$ of the needle were stripped of insulation and bent $30^{\circ}$ to form the probe. The barb electrode was made from 50 -stranded, 1/1,000 of an inch diameter, stainless steel lead wire (part No. A5635, 316LVM, Cooner Wire, Inc; Chatsworth, California) that is highly flexible and coated with Teflon ${ }^{\circledR}$. An 8 mm length of insulation was stripped from the wire, bent back as a barb, and placed in a

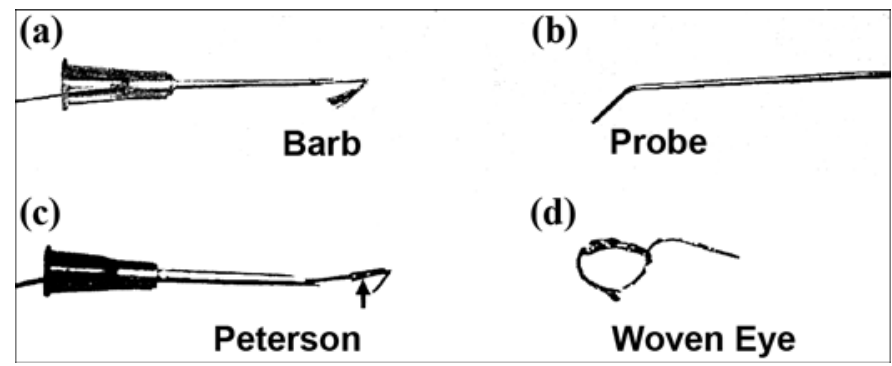

Figure 1.

Study electrodes. (a) Barb electrode with $8 \mathrm{~mm}$ exposed wire bent back and placed in 20-gauge needle. (b) Probe electrode with $8 \mathrm{~mm}$ exposed needle. (c) Peterson Intramuscular Electrode with $5 \mathrm{~mm}$ exposed wire and polypropylene barb placed in 18-gauge needle. Arrow points to middle of 10-piece polypropylene barb. This commercial electrode was not used in this study but is shown for comparison. (d) Woven eye electrode $1 \mathrm{~cm}$ in diameter. As reference, length of shanks of 20- and 18-gauge needles is $25 \mathrm{~mm}$.

20-gauge needle. On the end, distal to the electrode, a block fork crimp terminal (SPC Technology; Chicago, Illinois) was used for connection to a stimulator. The large surfacearea woven eye electrode was made from the same multistranded stainless steel lead wire as the barb electrode. The wire was stripped of $8 \mathrm{~cm}$ of insulation and woven into a circle that was $1 \mathrm{~cm}$ in diameter. All electrodes were autoclave sterilized.

We used an anal balloon to record pressure from a modified rectal balloon (Life-Tech Inc; Stafford, Texas) and placed Silastic adhesive (MED-1037, NuSil Technology; Carpinteria, California) on each end of the rectal balloon to help maintain the balloon in the anus. Stimulators (S48, Astro-Med, Inc; Houston, Texas) with constant current and stimulus isolation were used. Stimulating voltage and current were monitored on a 2-channel isolated oscilloscope (TekScope, Textronix; Beaverton, Oregon). Electrode resistance was determined based on Ohm's law. The current was determined by the voltage drop across a $100 \Omega$ resistor in series with the stimulating electrodes. For the stimulating voltage, an average voltage was used. The voltage from the stimulating electrodes sloped to a plateau, showing a capacity effect. With the use of an average voltage drop during the pulse, the capacity effect is included in the electrode resistance. Pressure recordings were made with WPI transducers (World Precision Instruments; Sarasota, Florida), connected to Gould amplifiers (Gould Instruments Systems Inc; Cleveland, Ohio) and recorded on an Astro-Med 8-channel recorder. 
The protocol received Institutional Animal Care and Use Committee and institutional approval, and five female dogs of moderate size were obtained from approved vendors. General anesthesia was induced with sodium thiopental anesthetic (2 $\mathrm{mg} / \mathrm{kg}$ intravenous dose) followed shortly by respiratory anesthetic (Isoflurane 2\%-3\%) for up to 4 hours. Animals were placed in supine position and prepped and draped in the usual sterile manner. An 8-inch midline incision was made from the caudal end of the pubic bone to the mid-abdominal area, and hemostasis was maintained with electrocautery. Blunt dissection was carried down to the perivesical fascia. Bilateral inferior neurovascular bundles, urethra, and bilateral ureters were identified, and adipose deposits were carefully dissected from the structures of interest. A balloon-tipped catheter (Cook urodynamics catheter, Cook Urological Co; Spencer, Indiana) was passed through the urethra into the bladder for filling and recording pressure. The balloon was filled and pulled against the bladder neck to prevent voiding. The bladder was filled to $45 \mathrm{~mL}$ for all stimulation studies except where different volumes were investigated.

Contraction of pelvic skeletal muscles was monitored in four ways. The first two consisted of observing the abdominal rectus and leg muscles and palpating for any movement. Third, the anal sphincter pressure was recorded with the anal balloon, and fourth, abdominal pressure was recorded with a rectal balloon in the abdomen for a more direct recording of abdominal muscle contraction. However, this pressure could only be recorded when the abdomen was closed during the final testing.

To limit the total number of stimulations and provide standard procedures, we used common protocols for probe, woven eye, and barb electrode testing. The common protocols included bilateral and bipolar electrode arrangements. The bipolar electrodes were approximately $8 \mathrm{~mm}$ apart and, where possible, were perpendicular to the pelvic nerves. The negative electrode was more caudal. The positive rostral electrode was usually over a branching point of the nerves on the bladder wall and thus was not perpendicular to all the branches. Common stimulation parameters were $40 \mathrm{pps}$ (pulses per second), $400 \mu$ s pulse duration, and a $3 \mathrm{~s}$ stimulation period. Current-response testing was conducted from 2 to $25 \mathrm{~mA}$. Alternatively, a single current of $20 \mathrm{~mA}$ was used for testing. This current was determined during the first series of testing in the first animal as a current that would induce a peak bladder pressure $>30 \mathrm{cmH}_{2} \mathrm{O}$ without skeletal muscle contraction. In one test, $10 \mathrm{~mA}$ had to be used because the $20 \mathrm{~mA}$ caused adverse leg contractions with one electrode arrangement.
Furthermore, to limit the total number of stimulations, we conducted single testing rather than duplicate testing but repeated the initial stimulating parameters in a series at the end of the series. Deviations from these common protocols are described in the following paragraphs.

Initial testing was done with the abdomen open, and we used the bilateral probe electrodes to determine an effective stimulation site. Three locations were tested (Figure 2). Site 1 was on the pelvic plexus nerves near the bladder wall. This location was next to or on the inferior neurovascular bundle, between the ureters and bladder neck. Test sites 2 and 3 were 1 and $2 \mathrm{~cm}$ more caudal along the bladder neck and urethra. A single $20 \mathrm{~mA}$ current was used in this comparison. An effective site was determined.

The next test at the effective site used bilateral probe electrodes and compared monopolar with bipolar stimulation. For monopolar stimulation, we used two distant positive electrodes that had $10 \mathrm{~cm}$ of exposed wire. These electrodes were placed under the skin along the midline of the chest, a location that does not cause skeletal muscle contraction. The negative electrodes were held next to the pelvic plexus nerves. For the bipolar arrangement, the common protocols were used with both the positive and negative electrodes held next to the pelvic nerve. We used the single current of $10 \mathrm{~mA}$ for this test because the $20 \mathrm{~mA}$ caused adverse leg contractions with one of the electrode arrangements. For all additional testing, we used the bipolar electrode configuration.

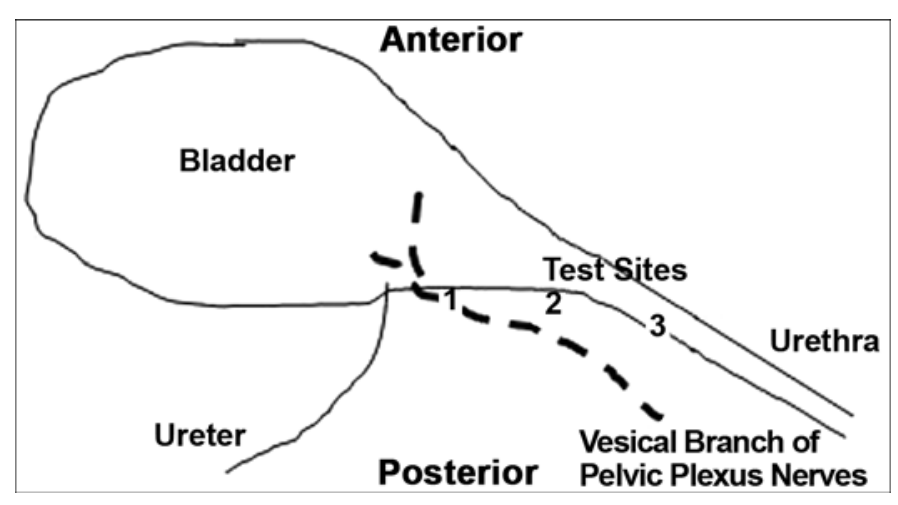

Figure 2.

Diagram of electrode stimulation test sites 1,2 , and 3 and their relationship to lower urinary tract, ureter, and pelvic nerve in five dogs. Site 1 is close to vesical nerve branch from pelvic plexus in neurovascular bundle, site 2 is $1 \mathrm{~cm}$ caudal, and site 3 is $2 \mathrm{~cm}$ caudal. Dashed line indicates pelvic plexus nerves in neurovascular bundle. Although unilateral stimulation sites are shown, most protocols used bilateral methods. 
Implantation of bilateral and bipolar barb electrodes was then conducted at the effective site next to the vesical nerve branch of the pelvic plexus. First, a needle holding the barb electrode was inserted into the fascia 1 to $2 \mathrm{~cm}$ lateral to the nerve and advanced until the electrode lay next to the nerve. Next, the needle was withdrawn and the electrode cable sutured to the fascial plane. Finally, bilateral current-response testing was conducted. The barb electrodes were reinserted if peak bladder pressures were lower than those obtained with the prior probe electrodes. Further comparisons of unilateral and bilateral stimulation were conducted at $20 \mathrm{~mA}$ stimulating current.

During each stage of the three-stage implantation process (probe, barb, current-response testing) just described, we also conducted unilateral testing on both sides of the bladder at $20 \mathrm{~mA}$. Additional testing was conducted with the closed abdomen and using the common protocols with the $20 \mathrm{~mA}$ stimulating current only. The final parameters investigated were pulse durations, stimulating frequencies, periods of stimulation, use of one stimulator that was connected to both sides of the bladder, and effects of bladder volume. Comparison of one stimulator with two stimulators for bilateral stimulation required special connections when the one stimulator was used. The one stimulator was connected in parallel to the implanted bilateral electrodes. With this one stimulator, we used $40 \mathrm{~mA}$, because a parallel circuit divides the current for approximately $20 \mathrm{~mA}$ to each side. Using a two-stimulator method, we connected one stimulator to each side of the bladder with $20 \mathrm{~mA}$ applied. We used about $45 \mathrm{~mL}$ filling volume for the volume test so that we could assess whether small changes in bladder volume could have affected the results of other studies. All results are shown as mean \pm standard deviation and paired Student's $t$-tests conducted with significance established at $p=0.05$; or not significant.

\section{RESULTS}

At test site 1 (Figure 2), one or two branches of the pelvic plexus nerves could be identified in the bladder inferior neurovascular bundle and the nerves were seen to branch near the bladder wall. Using the probe electrodes and common stimulation protocols (bilateral, bipolar, and $20 \mathrm{~mA}$ ), we induced the highest peak bladder contractions at this location. At sites 2 and 3, significantly lower pressures were recorded 1 and $2 \mathrm{~cm}$ away from the nerves and along the urethra (Figure 3). Adverse leg contractions were

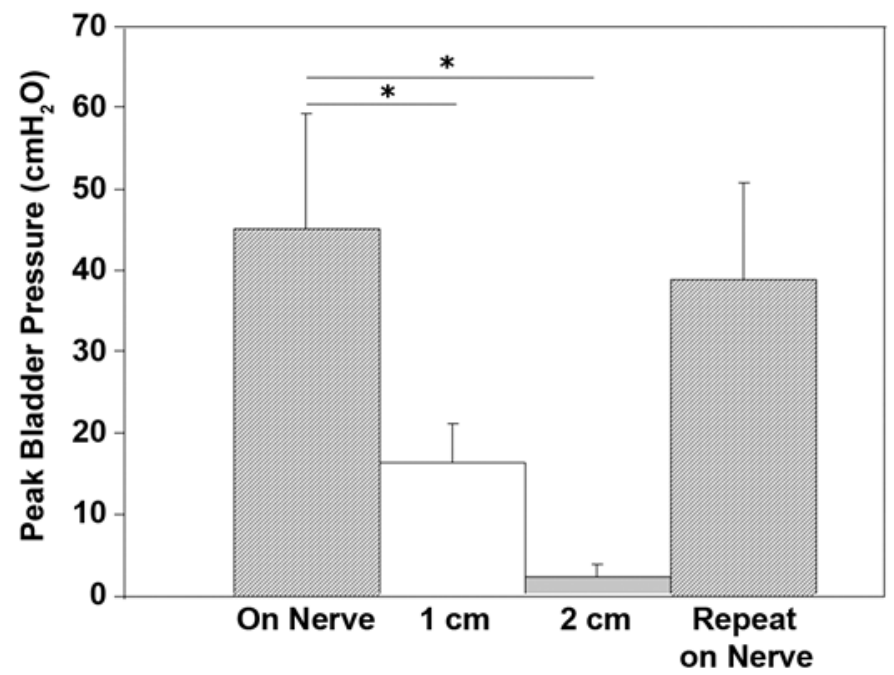

Figure 3.

Peak bladder pressure responses to stimulation with probe electrode close to pelvic plexus nerves in inferior neurovascular bundle and at 1 and $2 \mathrm{~cm}$ caudal in five dogs from single tests (see Figure 2 for location of 3 test sites). Stimulation was with common protocols, including $20 \mathrm{~mA}$. Unwanted leg contractions were only seen in two animals at $2 \mathrm{~cm}$ caudal location (site 3). *Error bars indicate standard deviation of mean.

also seen in two of the five animals at site 3 , the most caudal location. Thus further testing was done at site 1 only.

For the next test at site 1, we compared bipolar to monopolar stimulation and used the probe electrodes. Common stimulation protocols were used at a single current. In this comparison, $10 \mathrm{~mA}$ rather than $20 \mathrm{~mA}$ was used because monopolar stimulation caused adverse leg contractions at the higher current. Bipolar stimulation at this current induced a peak bladder pressure of $18.2 \pm$ $14.2 \mathrm{cmH}_{2} \mathrm{O}$, which was not significantly different than monopolar stimulation of $12.4 \pm 9.2 \mathrm{cmH}_{2} \mathrm{O}$. However, monopolar stimulation induced leg and anal sphincter contractions that were not seen with bipolar stimulation. The average anal pressure with monopolar stimulation was $16.4 \pm 23.4 \mathrm{cmH}_{2} \mathrm{O}$. Thus, for further testing, we used bipolar electrodes.

We then compared the large surface-area woven eye electrode with the probe electrode using the common stimulation protocols (bipolar and bilateral) at $20 \mathrm{~mA}$. The induced peak pressure for the probe was $37.5 \pm$ $13.6 \mathrm{cmH}_{2} \mathrm{O}$, which was significantly higher than the woven eye peak pressure of $19.8 \pm 11.7 \mathrm{cmH}_{2} \mathrm{O}$. No skeletal muscle contractions were noted with either the woven eye or probe electrode. 
The barb electrodes were then implanted. The first stage for implantation was to conduct current-response testing with the probe electrode. The second stage included implanting the barb electrode close to the nerves and conducting further testing (Figure 4). The needle holding the barb electrodes could easily be advanced in the fascia so that the electrode could be placed close to the pelvic nerve. One implanted electrode did not produce a bladder contraction when activated but did upon reimplantation. The final stage of implantation included closing the abdomen and conducting further currentresponse testing. No significant differences in induced peak bladder pressures were found for any of the currents tested for the three stages of implantation (Figure 5). Also, no skeletal muscle activation was observed during this testing, and no change in abdominal pressure occurred during stimulation after abdominal closure.

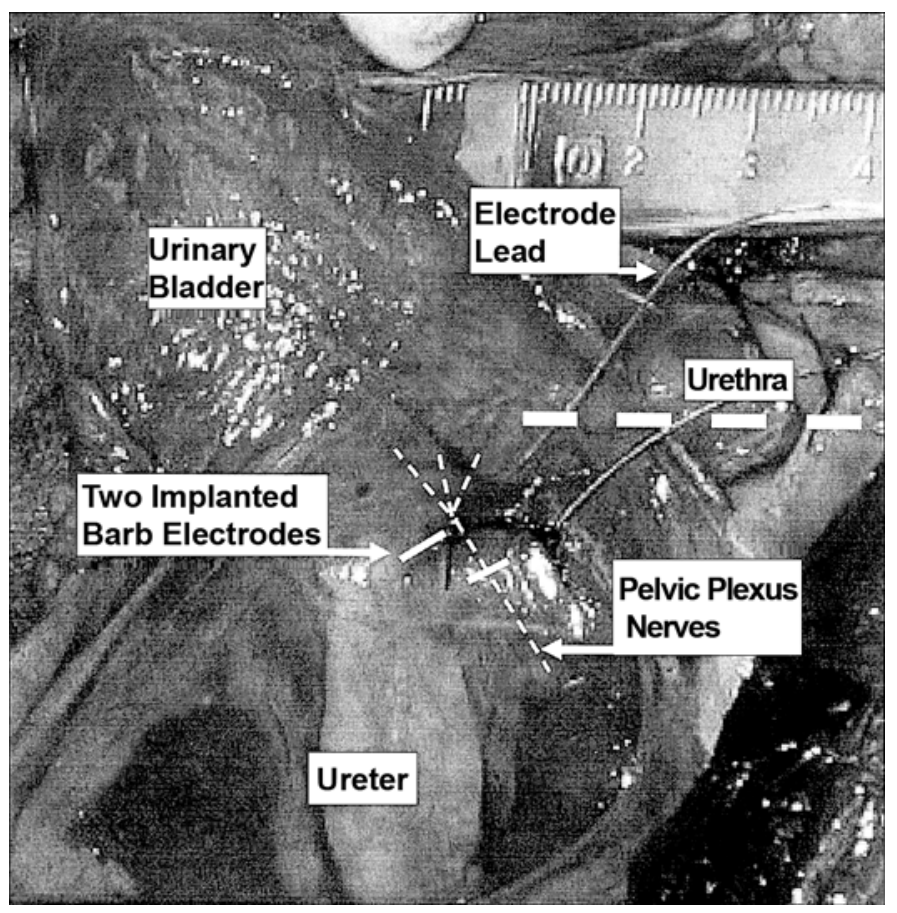

Figure 4.

Two barb electrodes implanted next to pelvic plexus nerves and adjacent to bladder wall and ureter in five dogs. Sutures are shown on electrode leads as they exit from fascia. Solid white lines indicate approximate location of electrodes under fascia, and narrow dashed white line is approximate location of pelvic plexus nerves with branches innervating bladder. Wide dashed line indicates middle of urethra. Fascia is spread and shows nerves more lateral from urethra. Centimeter ruler is shown at top right.
Unilateral testing (both right and left sides) was also conducted during the three stages of implantation. Comparison of stimulation between the right and left sides of the bladder did not result in a significant difference in peak pressures; thus the results from both sides were combined for comparison to bilateral stimulation. Unilateral stimulation at each stage of the implantations induced significantly lower peak bladder pressures than bilateral stimulation (Figure 6).

The final testing (third stage) was conducted with the abdomen closed and using common protocols (bilateral and bipolar) that included $20 \mathrm{~mA}$ stimulating current and the implanted barb electrodes. As shown in the Table, the induction of peak bladder pressures was again compared. Stimulation polarity of the implanted electrodes did not significantly affect the induced peak bladder pressures. Bilateral electrodes connected in parallel to one stimulator were compared to the standard two stimulators. The $40 \mathrm{~mA}$ applied from one stimulator induced a similar pressure to the two stimulators with $20 \mathrm{~mA}$. The

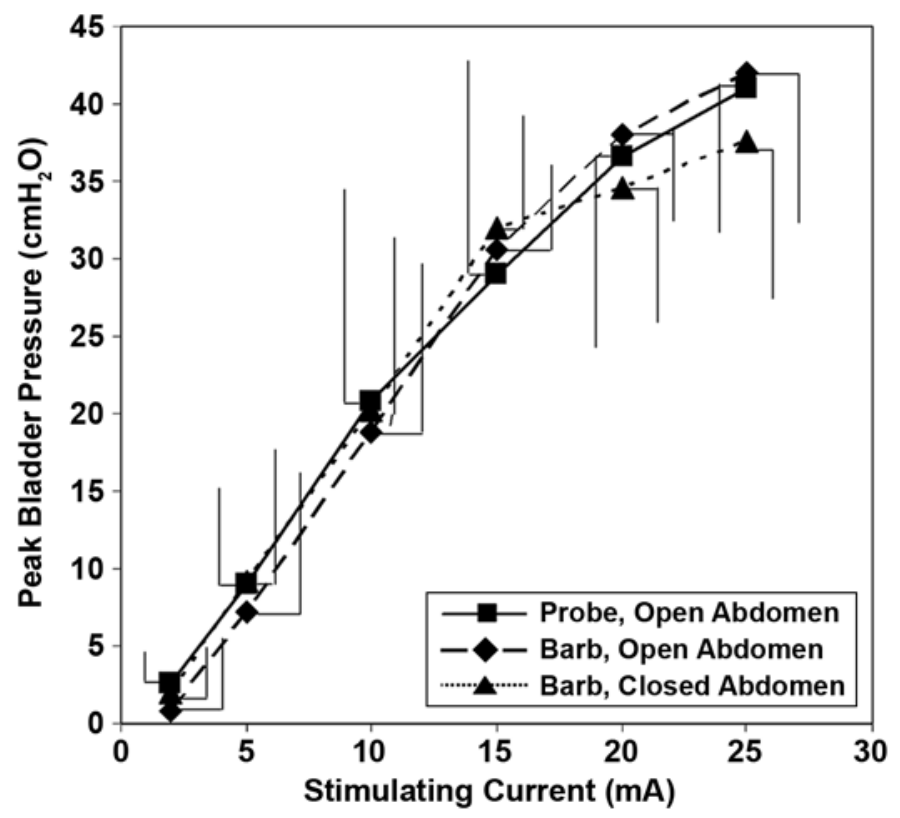

Figure 5.

Peak bladder pressure responses to stimulation during three stages of electrode implantation of five dogs. Stage 1: probe electrodes, stage 2: implanted barb electrodes and open abdomen, and stage 3: further testing with closed abdomen. Common stimulation protocols included bilateral, bipolar, 40 pps, 400 us pulse duration, and 3 s with currentresponse testing. Error bars indicate standard deviation of mean; no significant difference was found between three stages. 


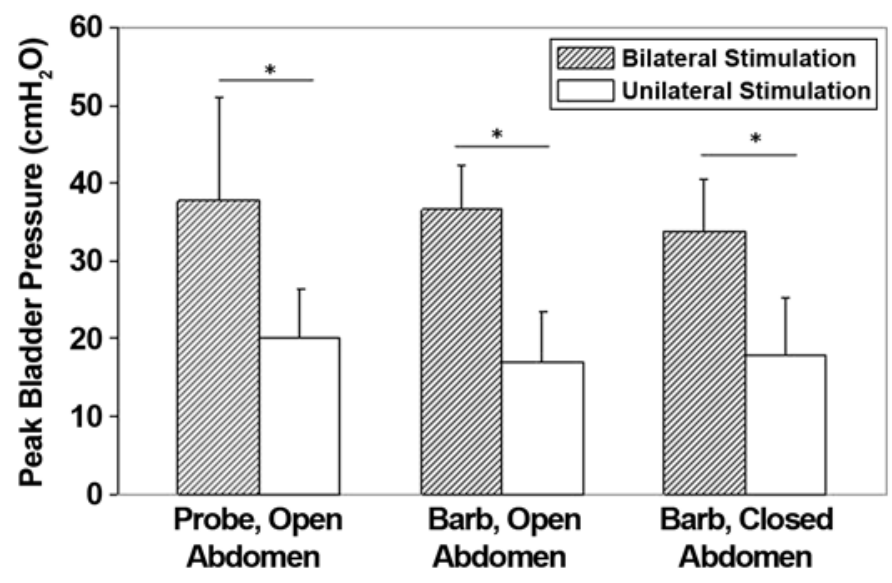

Figure 6.

Comparison of peak bladder pressure responses to bilateral and unilateral stimulation during three stages of electrode implantation in five dogs $(N=10$ [each side] for unilateral stimulation and 5 for bilateral stimulation). Common protocols used with $20 \mathrm{~mA}$. * Error bars indicate standard deviation of mean.

pulse duration of $400 \mu$ s only induced significantly higher peak pressures than $50 \mu \mathrm{s}$, and 40 pps stimulating frequency only induced significantly higher pressures than 10 pps. Finally, the Table compares the effects of bladder volumes from 25 to $60 \mathrm{~mL}$, and these different volumes did not significantly affect the peak pressure response to stimulation.

Two animals' bladders had spontaneous contractions without stimulation when filled to the standard volume of $45 \mathrm{~mL}$ (Figure 7(a) describes the test of one of these animals). The spontaneous contractions recorded in these animals had peak pressures in the range of those induced with high stimulating currents. Only one spontaneous contraction had a peak pressure higher than that induced with high-current electrical stimulation. This spontaneous response that was higher than the maximal stimulation-induced response is shown in Figure 7(a)-(b). In addition, in a few records, these same two animals showed continued bladder contractions with sustained pressure for 3 to $10 \mathrm{~s}$ following stimulation. Five sustained contractions occurred for one animal and three in the second animal. None of the other three animals had sustained bladder contractions following stimulation.

The total number of stimulations in the 2- to 3-hour recording sessions was $76 \pm 12$. All of these stimulations were for $3 \mathrm{~s}$ except for three stimulations for $10 \mathrm{~s}$. Three
Table.

Effects of polarity, number of stimulators, pulse duration, stimulating frequency, and bladder volume on induced peak bladder pressure during electrode bladder stimulation in five dogs.

\begin{tabular}{|c|c|}
\hline Variable & $\begin{array}{l}\text { Peak Bladder Pressure } \\
\left(\mathrm{cmH}_{2} \mathrm{O}\right)(\text { Mean } \pm \text { SD) }\end{array}$ \\
\hline \multicolumn{2}{|c|}{ Stimulation Polarity, NS $(n=5)^{*}$} \\
\hline Normal & $34 \pm 9$ \\
\hline Reverse & $32 \pm 6$ \\
\hline Normal & $35 \pm 6$ \\
\hline \multicolumn{2}{|c|}{ No. of Stimulators, NS $(n=3)^{\dagger}$} \\
\hline Two & $35 \pm 8$ \\
\hline One & $31 \pm 5$ \\
\hline \multicolumn{2}{|c|}{ Pulse Duration, $\mu$ s $(n=3-5)^{\ddagger}$} \\
\hline 400 & $34 \pm 6$ \\
\hline 200 & $29 \pm 7$ \\
\hline 100 & $21 \pm 12$ \\
\hline $50^{\S}$ & $17 \pm 12$ \\
\hline 400 & $34 \pm 7$ \\
\hline \multicolumn{2}{|c|}{ Stimulating Frequency, pps $(n=5)^{\Uparrow}$} \\
\hline 40 & $35 \pm 7$ \\
\hline 20 & $24 \pm 13$ \\
\hline $10^{* *}$ & $14 \pm 15$ \\
\hline \multicolumn{2}{|c|}{ Bladder Volume, mL, NS $(n=4)^{\dagger \dagger}$} \\
\hline 25 & $33 \pm 10$ \\
\hline 45 & $34 \pm 6$ \\
\hline 60 & $34 \pm 7$ \\
\hline $\begin{array}{l}\text { Note: All protocols used com } \\
\text { electrodes, } 40 \text { pps, } 400 \mu \mathrm{s}, 3 \mathrm{~s} \text {, } \\
{ }^{*} \text { Normal polarity of electrode } \\
\text { negative electrode at most e } \\
\text { effective location, and revers } \\
{ }^{\dagger} \text { Two stimulators each using } 20 \\
{ }^{\ddagger} \text { Compared pulse durations. } \\
{ }^{\S} \text { Significantly different from fi } \\
{ }^{\uparrow} \text { Effects of stimulating frequen } \\
{ }^{* *} \text { Significantly different from } \\
{ }^{\dagger} \text { Effects of bladder volume. } \\
\text { NS = not significant }(p=0.05)\end{array}$ & $\begin{array}{l}\text { n including bipolar and bilateral } \\
\text { cept where indicated. } \\
\text { in "Methods" section was with } \\
\text { and positive electrode at less } \\
\text { hed polarity. } \\
\text { with one stimulator using } 40 \mathrm{~mA} \text {. } \\
\text { with } 400 \mu \mathrm{s} \text {. } \\
10 \mathrm{~s} \text {. } \\
\text { deviation. }\end{array}$ \\
\hline
\end{tabular}

of the animals had more prolonged bladder contractions early in the recording session than at the end, whereas two animals had contractions of similar length throughout the session. Figure 7(b)-(d) shows one of the animals that developed shorter bladder contractions but with little change in peak pressure. Induced peak pressures were sustained throughout testing in the other animals. Finally, electrode resistance was determined for three bipolar arrangements with the implanted barb electrodes, which averaged $613 \pm 133$. 
(a)

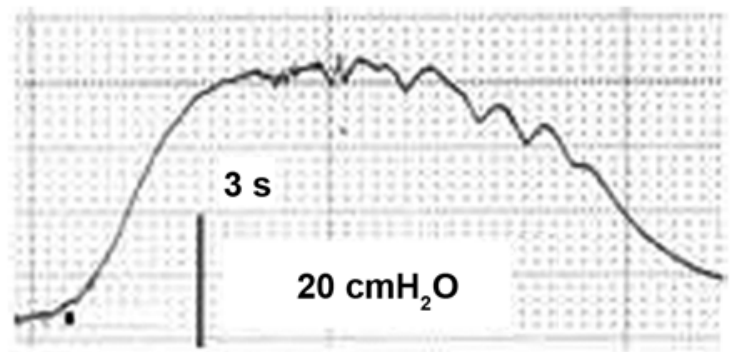

(b)

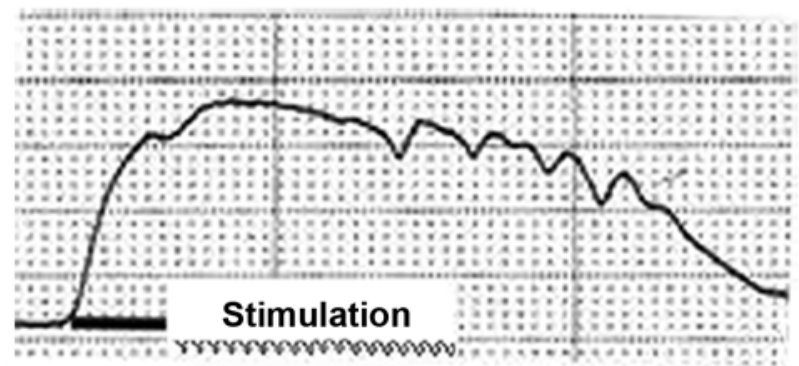

(c)

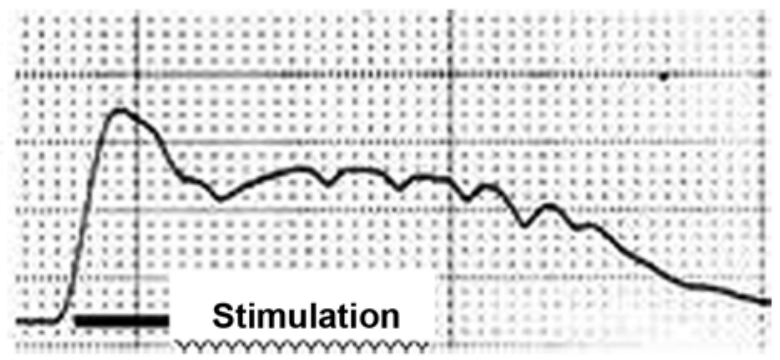

(d)

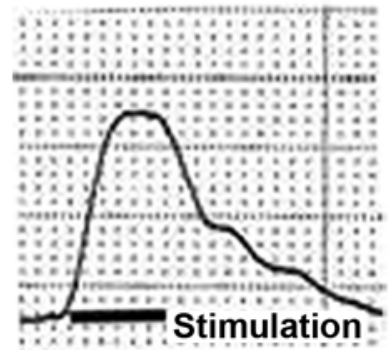

Figure 7.

Spontaneous bladder contraction and responses to stimulation in one dog over several hours of testing. (a) First spontaneous bladder contraction at start of recording. (b) Early stimulation-induced bladder contraction with bipolar probe electrodes. Continued bladder contraction following stimulation is shown. (c) Subsequent stimulation with implanted barb electrodes showing less sustained bladder contraction. (d) Following abdominal closure, again showing less sustained peak bladder pressure response to stimulation. Common stimulation included bipolar, bilateral, $40 \mathrm{pps}, 400 \mu$ s pulse duration, $3 \mathrm{~s}$, and $20 \mathrm{~mA}$.

\section{DISCUSSION}

Stimulation protocols were identified that induced bladder contractions without skeletal muscle activation. Selective procedures were neurosurgical techniques, electrode size and arrangements, and stimulation parameters. Important neurosurgical techniques included (1) visually identifying the nerves in the inferior neurovascular bundle and testing at that location, (2) implanting the barb electrode with a needle close to the nerve and testing, and (3) final testing following abdominal closure. The location next to the pelvic plexus nerves and neurovascular bundle was important because stimulation 1 or $2 \mathrm{~cm}$ more caudal along the urethra were less effective, and in a few animals, this location was associated with current spread and leg contractions.

The barb electrode and needle implantation methods were important for stimulation. The barb electrode performed better than the larger surface-area woven eye electrode. This result was probably because of the higher charge injection density at any given stimulating current with the barb electrode. The small size of the barb electrode also facilitated placement of the devices close together for bipolar stimulation. The bipolar arrangement was particularly important because monopolar stimulation resulted in current spread and unwanted skeletal muscle activation [19]. In contrast, in prior chronic animal studies, we reported that large surface-area electrodes, both the woven eye and suture electrodes, were effective for bladder stimulation [19-21]. However, these prior studies had important differences in the animal models used, such as bladder wall hypertrophy, SCI, and no use of anesthesia. Stimulation in these other models easily induced bladder contractions, and no comparison with smaller electrodes was conducted. Thus current observations probably show more effective methods of stimulation.

Prior direct bladder stimulation studies in patients with SCI may have used less than optimal stimulation methods. Most studies have used Avco (Avco Corp; Everett, Massachusetts) or Mentor (Mentor Corp; Santa Barbara, California) stimulators [9-13]. These stimulators used two or four long, flexible helical-wire (Helical Wire Inc; Wylie, Texas) electrodes that were sutured (imbricated) into the bladder wall. The various locations for these bipolar electrodes included ventral, mid-bladder wall, and bladder base. Problems reported in some patients included detrusor sphincter dyssynergia (high 
urethral resistance), poor voiding responses, leg contractions, and pain. Electrode migration was also a problem for some patients. More recently, Magasi et al. reported encouraging results with their 10 -year clinical experience [14-15]. Eight disk electrodes were sutured to the bladder wall. Implantation of two of the disks near the uretervesical junction was described as the most important location for inducing strong bladder contractions. This location corresponds to the inferior neurovascular bundle implantation site described here. The disk electrodes were effective in inducing bladder contractions and voiding in 29 of 32 patients. Of these patients, 21 had peripheral lesions and 11 had central neural lesions. In three patients with upper-motoneuron central lesions, transurethral bladder neck resection was required to reduce urethral resistance for effective stimulation-driven voiding. Surprisingly, five implants were removed because of infections, but these patients developed volitional voiding and bladder emptying following device removal. In view of all the clinical results, the spread of stimulating current may have been a problem due to less than optimal electrode size and locations. The large electrode surface area and wide separation of the electrodes are not optimal. In addition, systematic intraoperative testing to identify a low current stimulation site was often not done; placement was usually based on experience of where the pelvic nerves were expected to be. Thus alternative methods, as proposed here, may be considered to reduce the spread of current. A shortcoming of our intraoperative technique was that the different test locations were only tested at $20 \mathrm{~mA}$. In the future, investigators should use lower currents, including threshold currents, to better identify optimal stimulation sites.

Detrusor sphincter dyssynergia can occur in individuals following SCI. This high urethral resistance occurs when bladder contractions cause a reflex contraction of the urethral sphincters [1-3]. This resistance is a problem with direct bladder stimulation [7-8]. In addition, current spread with direct bladder stimulation can stimulate the pudendal nerve to cause urethral closure [7-8]. This adverse effect from the pudendal nerve could occur in several ways, e.g., direct activation of motor fibers and/or sensory fiber stimulation with reflex motor nerve activation. Two sphincters are found in the urethra: a proximal smooth-muscle sphincter and a distal skeletal muscle sphincter; both can have unwanted closure. Skeletal sphincter closure is considered the most serious $[11,13]$. The current animal studies are limited in evaluating these different urethral closure mechanisms. The use of anesthesia limits spinal reflex activity. Because SCI was not conducted, reflex detrusor sphincter dyssynergia could not be evaluated. Urethral sphincter contractions were not recorded. The balloon catheter in these studies was pulled against the bladder neck to prevent pressure recording or voiding. The quadruped animal model may also be limited. The bladder in dogs is located rostral in the abdomen, whereas in humans, the bladder is directly above the pelvic floor. Thus current spread is less likely to activate the pudendal nerve in this animal model than in humans [20]. Important methods that were used included monitoring contractions of the anal sphincter, pelvic floor, abdomen, and legs. Because none of these muscles contracted during stimulation of the pelvic plexus with bipolar electrodes at the highest current of $25 \mathrm{~mA}$, this finding indirectly shows that the urethral skeletal sphincter was not activated. Future studies of direct bladder stimulation should include voiding and direct urethral pressure-monitoring methods [11].

With the abdomen closed, we compared different stimulating parameters. Common stimulating parameters to induce peak bladder pressures of 30 to $40 \mathrm{cmH}_{2} \mathrm{O}$ were identified as $40 \mathrm{pps}, 15$ to $25 \mathrm{~mA}, 400 \mu$ s pulse duration, and $3 \mathrm{~s}$ of stimulation. Lower stimulating currents, frequencies, and shorter pulse durations were less effective in inducing high peak pressures (Table). We reported similar stimulating parameters for direct bladder stimulation in chronic cat models [19-23]. However, the induced peak pressures in those animals were higher [19-20,2223]. The higher pressures may have resulted from studies without anesthesia (not needed after SCI) and a model with bladder wall hypertrophy. Using a different approach to limit the spread of current, Staubitz et al. used monopolar cuff electrodes on the pelvic plexus nerves in acute and chronic dogs [24]. The cuff was applied a few centimeters caudal to the bladder neck, and stimulation in the acute dogs at $1.5 \mathrm{~V}$ and 20 pps induced peak bladder pressure from 33 to $59 \mathrm{cmH}_{2} \mathrm{O}$. Higher pressures were induced at $3 \mathrm{~V}$, but skeletal muscle contraction occurred. Results in their chronic dogs also showed higher peak bladder pressures, but these results were attributed to bladder wall hypertrophy. Use of pelvic plexus nerve-cuff electrodes presents two concerns: difficult surgical implantation and possible nerve injury.

We also tested unique methods to reduce current spread or the number of stimulators. Unilateral stimulation was tested during all three stages of the implantation 
process. Peak bladder pressures were always less than those induced with bilateral stimulation. The use of one stimulator instead of two for bilateral stimulation was also tested. Although one stimulator in a parallel circuit with the bipolar and bilateral electrodes was as effective as two stimulators, higher currents were required from the single stimulator. These methods warrant further investigation because the number of implanted electrodes or stimulators needs to be limited.

Different animal models of urinary retention have been used for bladder stimulation studies [8,20-21]. Ebner et al. showed that direct bladder stimulation with intravesical electrodes was mediated through sensory nerves and activation of micturition reflexes. In our acute model with respiratory anesthetic and partial suppression of bladder reflexes, stimulation of pelvic plexus motor nerves is important [25]. Suppression of bladder reflexes was indicated by the decline in peak bladder pressure immediately following most stimulations. However, in the few cases of spontaneous bladder contractions or continued high-pressure contractions following the end of stimulations, suppression of bladder reflexes was shown to be limited. Another concern was that the results may have been influenced by the large number of stimulations that were conducted over the 2 to 3 hours of anesthesia. This large amount may not have been a problem because the induced peak bladder pressures remained high over this time period, showing that multiple testing can be conducted (Figure 7). In addition, the design of repeating the first test again at the end of each series showed repeatable results (Figure 3).

SCI with lesions above the sacral spinal level results in urinary retention, and direct bladder stimulation with a barb electrode is being investigated to induce bladder emptying. The Peterson Intramuscular Electrode is a barb electrode (see "Methods" section) that is used clinically to stimulate paralyzed muscles and could be considered for bladder stimulation [17]. However, we must point out that other lower urinary tract problems, in addition to urinary retention, need to be addressed for direct bladder stimulation to be effective. These problems are detrusor sphincter dyssynergia and urinary incontinence from an overactive bladder [1-3]. The high urethral resistance or detrusor sphincter dyssynergia problem was just explained, and botulinum toxin injection into the two urethral sphincters is a promising approach for preventing these unwanted contractions [26]. In addition, for the proximal smoothmuscle sphincter, the use of oral alpha-blocker medica- tion can be used for inhibition [27]. The urinary incontinence from an overactive bladder could be managed by oral anticholinergic medication or low doses of botulinum toxin into the bladder wall [1,28-29]. Although the peak bladder pressure induced with stimulation could be reduced by these treatments, other overactive bladder conditions are treated with these interventions without producing urinary retention.

The Ontario Neurotrauma Foundation has recently highlighted the need for new electrical stimulation methods for micturition control following SCI [30]. Pelvic plexus nerve stimulation close to the bladder wall, as a refined method of direct bladder stimulation, may be such an approach. Features of this method include the use of barb electrodes that could be implanted with minimally invasive methods and with the possibility of inducing sustained bladder contractions important for voiding. However, current spread and high urethral resistance need to be limited for this approach to be effective.

\section{CONCLUSIONS}

In this anesthetized animal model, the combination of identification of the pelvic plexus nerves in the inferior vesical neurovascular bundle and intraoperative testing and implantation was shown to be an effective neurosurgical technique. Implanting the barb electrodes with a needle in a bilateral and bipolar configuration was also shown to be effective. Common stimulation parameters included 15 to $25 \mathrm{~mA}, 40 \mathrm{pps}$, and a $400 \mu$ s pulse duration applied for $3 \mathrm{~s}$. These methods avoided the spread of the electrical current to leg and other skeletal muscles. However, voiding studies were not conducted that would have more directly shown the effects of stimulation on the urethral sphincters. Devices and neurosurgical methods evaluated here are important because they could be translated into clinical applications with minimally invasive laparoscopic techniques.

\section{ACKNOWLEDGMENTS}

This material was based on work supported by the Department of Veterans, Rehabilitation Research and Development Service merit review grant B3254R.

The authors have declared that no competing interests exist. 


\section{REFERENCES}

1. Consortium for Spinal Cord Medicine. Bladder management for adults with spinal cord injury: A clinical practice guideline for health-care providers. Washington (DC): Paralyzed Veterans of America; 2006.

2. Yalla SV, Fam BA. Spinal cord injury. In: Krane RJ, Siroky MB, editors. Clinical neuro-urology. 2nd ed. Boston (MA): Little Brown; 1991. p. 319-32.

3. Rudy DC, Awad SA, Downie JW. External sphincter dyssynergia: An abnormal continence reflex. J Urol. 1988; 140(1):105-10. [PMID: 3379672]

4. Johnson TM 2nd, Ouslander JG. Urinary incontinence in the older man. Med Clin North Am. 1999;83(3):1247-66. [PMID: 10503063]

5. Brindley GS. The first 500 patients with sacral anterior root stimulator implants: General description. Paraplegia. 1994; 32(12):795-805. [PMID: 7708419$]$

6. Creasey GH, Grill JH, Korsten M, Sang H, Betz R, Anderson, R, Walter J; Implanted Neuroprosthesis Research Group. An implantable neuroprosthesis for restoring bladder and bowel control to patients with spinal cord injuries: A multicenter trial. Arch Phys Med Rehabil. 2001;82(11): 1512-19. [PMID: 11689969]

7. Rijkhoff NJ, Wijkstra H, van Kerrebroeck PE, Debruyne FM. Urinary bladder control by electrical stimulation: Review of electrical stimulation techniques in spinal cord injury. Neurourol Urodyn. 1997;16(1):39-53.

[PMID: 9021789]

8. Van Balken M, Vergunst H, Bemelmans BL. The use of electrical devices for the treatment of bladder dysfunction: A review of methods. J Urol. 2004;172(3):846-51. [PMID: 15310981]

9. Hald T, Meier W, Khalili A, Agrawal G, Benton JG, Kantrowitz A. Clinical experience with a radio-linked bladder stimulator. J Urol. 1967;97(1):73-78. [PMID: 6016213]

10. Halverstadt DB, Parry WL. Electrical stimulation of the human bladder: 9 years later. J Urol. 1975;113(3):341-44. [PMID: 1117500]

11. Jonas U, Hohenfellner P. Later results of bladder stimulation in 11 patients: Follow-up to 4 years. J Urol. 1978; 120(5):565-68. [PMID: 309513$]$

12. Merrill DC, Conway CJ. Clinical experiences with the Mentor bladder stimulator. I. Patients with upper motor neuron lesions. J Urol. 1974;112(1):52-56. [PMID: 4835078]

13. Stenberg CC, Burnette HW, Bunts RC. Electrical stimulation of human neurogenic bladders: Experience with 4 patients. J Urol. 1967;97(1):79-84. [PMID: 6016214]

14. Magasi P, Simon Z. Electrical stimulation of the bladder and gravidity. Urol Int. 1986;41(4):241-45. [PMID: 3491454]
15. Magasi P, Novoszel T, Koncz P. Electronic bladder stimulation in spinal cord paralysis. Urol Int. 1976;31(5):332-42. [PMID: 1087493]

16. Memberg WD, Peckham PH, Keith MW. A surgicallyimplanted intramuscular electrode for an implantable neuromuscular stimulation system. IEEE Trans Rehabil Eng. 1994;2(2):80-91.

17. Onders RP, Elmo MJ, Ignagni AR. Home-based ventilator weaning in tetraplegia: Results of the diaphragm pacing stimulation system [abstract]. J Spinal Cord Med. 2006;29: 249-50.

18. Walter JS, Sidarous R, Robinson CJ, Wheeler JS, Wurster $\mathrm{RD}$. Comparison of direct bladder and sacral nerve stimulation in spinal cats. J Rehabil Res Dev. 1992;29(2):13-22. [PMID: 1578388]

19. Walter JS, Wheeler JS, Cogan SF, Plishka M, Riedy LW, Wurster RD. Evaluation of direct bladder stimulation with stainless steel woven eye electrodes. J Urol. 1993;150(6): 1990-96. [PMID: 8230551]

20. Walter JS, Wheeler JS, Cai W, Wurster RD. Direct bladder stimulation with suture electrodes promotes voiding in a spinal animal model: A technical report. J Rehabil Res Dev. 1997;34(1):72-81. [PMID: 9021627]

21. Walter JS, Wheeler JS, Cai W, King WW, Wurster RD. Evaluation of a suture electrode for direct bladder stimulation in a lower motor neuron lesioned animal model. IEEE Trans Rehabil Eng. 1999;7(2):159-66. [PMID: 10391586]

22. Walter JS, Wheeler JS, Fitzgerald MP, McDonnell A, Wurster RD. A spinal cord injured animal model of lower urinary tract function: Observations using direct bladder and pelvic plexus stimulation with model microstimulators. J Spinal Cord Med. 2005;28(3):246-54. [PMID: 16048143]

23. Walter JS, Fitzgerald MP, Wheeler JS, Orris B, McDonnell A, Wurster RD. Bladder-wall and pelvic-plexus stimulation with model microstimulators: Preliminary observations. J Rehabil Res Dev. 2005;42(2):251-60. [PMID: 15944889]

24. Staubitz WJ, Cheng SF, Gillen HW, Holmquest B, Zurlo P, Greatbatch W. Management of neurogenic bladder in paraplegic dogs by electrical stimulation of pelvic nerves. Invest Urol. 1966;4(1):20-29. [PMID: 5941033]

25. Ebner A, Jiang C, Lindstrom S. Intravesical electrical stimulation-An experimental analysis of the mechanism of action. J Urol. 1992;148(3):920-24. [PMID: 1512860]

26. Wheeler JS Jr, Walter JS, Chintam RS, Rao S. Botulinum toxin injections for voiding dysfunction following SCI. J Spinal Cord Med. 1998;21(3):227-29. [PMID: 9863933]

27. Linsenmeyer TA, Horton J, Benevento J. Impact of alpha 1-blockers in men with spinal cord injury and upper tract stasis. J Spinal Cord Med. 2002;25(2):124-28.

[PMID: 12137216] 
28. Cohen BL, Rivera R, Barboglio P, Gousse A. Safety and tolerability of sedation-free flexible cystoscopy for intradetrusor botulinum toxin-A injection. J Urol. 2007;177(3): 1006-10. [PMID: 17296397]

29. MacDiarmid SA. Maximizing anticholinergic therapy for overactive bladder: Has the ceiling been reached? BJU Int. 2007;99 Suppl 3:8-12. [PMID: 17488367]

30. Hayes KC, Bassett-Spiers K, Das R, Ethans KD, Kagan C, Kramer JL, Linsenmeyer T, Moore KN, Razvi H, Reid G,
Walter JS, Wilson JW. Research priorities for urological care following spinal cord injury: Recommendations of an expert panel. Can J Urol. 2007;14(1):3416-23.

[PMID: 17324320]

Submitted for publication April 18, 2007. Accepted in revised form October 5, 2007. 